\title{
Risiko- und Schadensminimierung - wie wirksam sind sie?
}

\section{A. Uchtenhagen}

\author{
Risk- and Harm Minimisation - How Effective Are They?
}

\section{Zusammenfassung}

Der Forschungsstand zur Frage von Legitimation und Zukunft von schadensmindernden Strategien bei an sich unerwünschtem Verhalten wird diskutiert. Thematisch handelt es sich um Safer Use bei intravenösem Drogenkonsum, Strategien zur Prophylaxe bei Freizeitdrogenkonsum, Schadensminimierung durch Suchtmittelersatz, Safer Sex und weitere Maßnahmen zur Optimierung von Schadensminderung. Eine Integration wirksamer Maßnahmen in nationale Drogenpolitik ist möglich und wünschenswert, diese stehen nicht im Widerspruch zu Prävention und Behandlung. Für negative Auswirkungen von Schadensminimierung gibt es kaum Belege, Verbesserungen scheinen dennoch nötig.

\section{Schliuisselwörter}

Schadensminderung • Risikominimierung • Drogenpolitik • Prophylaxe · Drogenkonsum · Safer Use

\section{Abstract}

The research evidence for a legitimacy and for options of harm reduction strategies in the field of undesirable risk behavior is presented. This concerns safer use in injecting drug consumption, strategies to minimize harm in leisure time drug use, harm reduction through substitution treatments, safer sex and other measures for optimising harm reduction in a Public Health and and Public Order perspective. An intergration of such strategies into a comprehensive drug policy is feasible and recommendable, as they are not in contrast to prevention and treatment. Evidence for negative effects of harm reduction measures is scarce. Nevertheless, improvements are needed.

Key words

Harm reduction · risk minimizing · drug policy · drug use · safer use

\section{Einleitung}

Schadensminderung ist seit der Aids-Epidemie ein integrierender Bestandteil der Drogenhilfe, wenn auch in einigen Aspekten und in einigen Ländern nach wie vor umstritten oder abgelehnt. In Europa bildet sie ein Element des „Action Plan on Drugs“ seit 1999 und ist auch für die Nachfolgeplanung für die Jahre 2005 bis 2009 festgeschrieben. Trotzdem begegnen ihr viele Menschen mit einer Ambivalenz, die der Klärung bedarf.
In der Sache ist der Grundsatz, Schaden abzuwenden, so alt wie die Medizin und bereits bei Hippokrates verankert. In vielen Bereichen, die mit der Gefährdung und Erhaltung der Gesundheit zu tun haben, ist der Grundsatz eine Selbstverständlichkeit und höchstens dort umstritten, wo er wirtschaftliche Interessen berührt. Das reicht von Lebensmittelgesetzgebung, Medikamentenkontrolle, Hygienevorschriften bis zur Helm- bzw. Gurttragepflicht und anderen Vorschriften zur Unfallverhütung in Sport und Alltag. Das Besondere an diesen Beispielen ist, dass es sich um Maßnahmen handelt, die die negativen Auswirkungen eines gesellschaftlich akzeptierten und legitimierten Verhaltens betreffen. 
Ganz anders ist die Lage bei unerwünschtem oder verbotenem Verhalten wie illegalem Drogenkonsum oder außerehelichem Geschlechtsverkehr. Hier geraten schadensmindernde Maßnahmen durch die Verminderung von negativen Folgen, von denen eine abschreckende Wirkung erhofft wird, in den Verdacht, dem unerwünschten Verhalten Vorschub zu leisten. „Harm reduction“ gerät in den Verdacht einer „harm production“. Eine besondere Stellung haben Verhaltensweisen, die zwar an sich gesellschaftlich akzeptiert sind - wie Alkohol- und Tabakkonsum oder Glücksspiel - deren exzessive Formen hingegen stigmatisiert sind.

Legitimation und Zukunft von schadensmindernden Strategien bei an sich unerwünschtem Verhalten stehen deshalb unter einem besonderen Erwartungsdruck: den Nachweis zu erbringen, dass sie tatsächlich mehr Schaden verhindern als Schaden stiften. Das soll hier aufgrund des gegenwärtigen Forschungsstands dargestellt und diskutiert werden.

\section{Begriffliche Klärung}

Schadensminderung betrifft die Vermeidung oder Verminderung von gesundheitlichen und/oder sozialen Schäden für das Individuum sowie für die Gemeinschaft (reducing morbidity and mortality in a public health perspective, and reducing nuisance in a public order perspective).

Schadensminderung ist nicht gleichbedeutend mit Legalisierung oder „Normalisierung“ eines gesellschaftlich abgelehnten oder verbotenen Verhaltens. Schadensminderung als drogenpolitisches Postulat ist nicht identisch mit dem Postulat einer Drogenlegalisierung. Legalisierung einer Droge kann nur dann als schadensmindernde Maßnahme gelten, wenn ihre Auswirkungen tatsächlich mehr Schaden verhüten als stiften. Um eine Überprüfbarkeit ihrer Auswirkungen zu erlauben, muss jede schadensmindernde Maßnahme definieren, welche Schäden sie vermindern soll und was ihre konkreten Ziele sind, die sie erreichen will.

Schadensminderung beruht in der Regel auf einer Verminderung von Risikoverhalten. Sie muss deshalb voraussetzen, dass sich das entsprechende Risikoverhalten ändern lässt und dass das veränderte Risikoverhalten tatsächlich zu einer Schadensminderung führt. Vorsichtiger ist es deshalb, von Risikominderung zu sprechen, solange ein Nachweis verminderter Schäden nicht erbracht ist.

\section{Strategien der Risikominderung}

Hier stehen vor allem die folgenden Strategien zur Diskussion:

- Strategien der Morbiditäts- und Mortalitätsprophylaxe bei intravenösem Drogenkonsum (Safer Use);

- Strategien der Mortalitäts- und Morbiditätsprophylaxe bei Freizeitdrogenkonsum;

- Schadensminderung durch Suchtmittelersatz;

- Schadensminderung durch geschützten Geschlechtsverkehr (Safer Sex) sowie

- weitere unterstützende Maßnahmen zur Optimierung einer Schadensminderung.
Abschließend soll das Beispiel einer integrierten Politik der Schadensminderung diskutiert werden.

Strategie der Morbiditäts- und Mortalitätsprophylaxe bei i. v. Drogenkonsum

Hier stehen zwei sich ergänzende Anliegen im Vordergrund:

- Verhinderung des Erwerbs und der Übertragung von Infektionen (HIV, Hepatitiden) durch kontaminiertes Spritzenmaterial; dazu eignen sich Spritzenumtauschprogramme (needle exchange programmes - NEP, syringe exchange programmes - SEP) und in begrenzterem Ausmaß Desinfektionsmittel für Spritzenmaterial (bleach) sowie

- Verhinderung von unhygienischen Injektionen und letalen Überdosierungen durch Überwachung und medizinische Hilfe in dafür geeigneten Injektionsräumen (safe injection rooms SIR, safe injection sites - SIS).

\section{Spritzenumtauschprogramme Zielsetzung}

Im Mittelpunkt dieser Überlegung steht die Bemühung, die Wiederverwendung gebrauchten Spritzenmaterials zu verhindern. Dieses wird gegen sterile Spritzen und Nadeln umgetauscht, mit oder ohne Beratungsangebot. Damit soll die Verbreitung von Infektionen, die auf dem Blutweg übertragen werden, eingeschränkt und zudem die Anzahl gebrauchter Spritzen, die im öffentlichen Raum herumliegen, vermindert werden.

Spritzenumtausch wird in verschiedenen Settings praktiziert: in speziellen Abgabestellen, in Drogenberatungsstellen und anderen Einrichtungen der Drogenhilfe, in Apotheken, in Automaten.

Ausgewertete Erfahrungen belegen die folgenden Auswirkungen:

- weniger Wiederverwendung gebrauchter Spritzen und weniger gemeinsame Verwendung von Spritzen [1 -3],

- positive Erfahrungen mit Spritzentauschprogrammen im Gefängnis [4-6], auch bei Aufstellung von Spritzenautomaten [7],

- niedrige Inzidenz von durch Blut übertragenen Infektionen [1, 5, 8-10],

- weniger gebrauchte Spritzen im öffentlichen Raum [1, 11, 12],

- niedrigere Serokonversionsraten in Städten mit Spritzentauschprogrammen im Vergleich zu anderen Städten [13],

- nicht mehr, sondern weniger Injektionen [1,14],

- hohe Bereitschaft, den Drogenkonsum zu reduzieren [15],

- Übertritte in drogenfreie Therapie oder in Methadonbehandlung $[14,16,17]$ sowie

- weniger Risikoverhalten und Behandlungsabbrüche bei ehemaligen Teilnehmern von Spritzentauschprogrammen, die sich in eine Therapie begeben [18].

Weitere Erfahrungen betreffen die Möglichkeit, Teilnehmer an einem Spritzentauschprogramm für eine begleitende Therapie zu gewinnen. So lässt sich durch motivationsfördernde Interventionen (motivational interviewing) der problematische Alkoholkonsum bei Drogenabhängigen reduzieren [19], was umso wichtiger erscheint, als unter Alkohol riskantes Injektionsverhalten häufig ist [20]. Oder die Anwendung verhaltenstherapeutischer 
Kurzinterventionen kann den Stimulanzienkonsum vermindern [21], ebenfalls von Bedeutung angesichts des deutlich häufigeren „needle sharing“ bei Kokainkonsumenten [22]. Die Teilnahme am Spritzentauschprogramm kann außerdem für Hepatitistests genutzt werden [23].

Dass die Erhältlichkeit von Spritzen allein oftmals nicht genügt für eine Verhaltensänderung und dass die Teilnahme an einem Spritzentauschprogramm zu einer Verminderung des Wiedergebrauchs von Spritzen führt, aber nicht notwendigerweise zu einem Verzicht auf den gemeinsamen Gebrauch von Spritzen, geht aus einer neueren Untersuchung hervor [24]. Eine aktive Beratung ist in vielen Fällen für einen Erfolg der Maßnahme erforderlich.

Wer nutzt die verschiedenen Angebote zum Umtausch gebrauchter Spritzen und Nadeln? Neben Opiatabhängigen und Abhängigen von Stimulanzien sind es zunehmend auch Personen, die sich anabole Steroide injizieren [25]. Wie aus einer französischen Studie aus Marseille hervorgeht, werden Spritzenautomaten häufiger von jüngeren, nicht substituierten Drogenabhängigen genutzt, im Vergleich zu den Umtauschprogrammen und den Apotheken mit Spritzenabgabe. Eine entsprechende Diversifizierung der Angebote wird deshalb als wichtig erachtet [26]. Eine norwegische Studie fand, dass die Häufigkeit der Benutzung eines Spritzenumtauschprogramms lediglich mit der Injektionshäufigkeit korreliert; die Wiederverwendung von Spritzen nahm im Laufe der Zeit deutlich ab, kam aber immer noch bei $51 \%$ der Programmbenutzer vor [1]. In Vancouver wurde festgestellt, dass "needle sharing“ trotz Spritzentauschprogrammen vor allem bei Personen persistierte, die zu weit weg von den Angabestellen lebten [27]. Psychiatrische Störungen erhöhen ebenfalls die Wahrscheinlichkeit riskanter Praktiken trotz vorhandenem Risikobewusstsein [28].

Die Teilnahme an einem Spritzentauschprogramm schließt aber nicht alle Infektionsrisiken aus. Infektionen können auch beim Gebrauch steriler Spritzen übertragen werden. Ein bekanntes Beispiel ist die gemeinsame Benutzung einer aus Opiumstroh hergestellten Opiatlösung (so genanntes Compot) in Osteuropa, aus der Aids- und Hepatitisviren aufgenommen werden. Ein anderes Beispiel ist die häufige Verwendung von ungeeigneten Filtern beim Aufziehen der Injektionslösung (ungeeignet für das Ausfiltern bakterieller Verunreinigungen, [29]). Und schließlich kann die Praxis des Frontloadings (Aufziehen der Opiatlösung aus einer anderen Spritze) zu einer Infektion führen [30].

Die Befürchtung, Spritzentauschprogramme könnten zu einem Anstieg intravenösen Drogengebrauchs führen, hat sich nicht bestätigt $[8,9]$.

Eine Analyse von 200 Studien zum Thema bestätigt die genannten positiven Erfahrungen sowie das Fehlen nachweisbarer negativer Auswirkungen [31].

\section{Spritzendesinfektion Zielsetzung}

Eine risikofreie Wiederverwendung soll durch Desinfektionsverfahren von Spritzenmaterial erreicht werden. Zur Anwendung gelangen Alkohol und im Haushalt verwendete Desinfektions- mittel wie Javelwasser o. ä. Dasselbe soll durch Auskochen erreicht werden. Die Durchführung solcher Maßnahmen ist häufig ungenügend. Insbesondere bieten sie zu wenig Schutz vor einer Infektion mit Hepatitis-C-Viren [32, 33].

\section{Injektionsräume Zielsetzung}

Die Einrichtung von Räumen, wo unter hygienischen Verhältnissen und professioneller Überwachung mitgebrachte Drogen injiziert werden können, soll eine Verminderung von Spritzeninfektionen und letalen Überdosierungen erlauben und außerdem zu einer Verminderung von Injektionen im öffentlichen Raum beitragen. Injektionsräume gibt es in der Schweiz seit 1986, in Deutschland seit 1994, in Spanien seit 2000. 2004 wurden in 36 europäischen Städten ca. 60 Injektionsräume gezählt [34]. Allein in Deutschland stieg die Zahl der dokumentierten Injektionen in Injektionsräumen von ca. 5000 im Jahr 1995 auf ca. 40000 im Jahr 2001 [34].

Injektionsräume werden nicht nur von Opiatabhängigen genutzt. In Vancouver wurde eine hohe Bereitschaft von Kokainabhängigen zur Benutzung von Injektionsräumen festgestellt [35].

Ausgewertete Erfahrungen sind zusammengefasst bei Hedrich 2004 [34]; sie betreffen u. a. die folgenden Auswirkungen:

- verbessertes Risikobewusstsein und vermindertes Risikoverhalten bei Benutzern, weniger Spritzeninfektionen, weniger Todesfälle durch Überdosis in den Standortstädten (wenn Angebot ausreichend) [36 - 39];

- erhöhte Bereitschaft für freiwillige Serotests und für Übertritt in eine Therapie (Review bei Broadhead [12]) sowie

- weniger Konsum in der Öffentlichkeit, mehr Nutzung der medizinischen und sozialen Dienstleistungen einschließlich Suchttherapien.

- Weitere Übersichtsarbeiten:

- Fischer et al. 2002 [40]: Überdosierungen (ca. 1 pro 500 bis 600 Injektionen) werden vor Ort behandelt, keine Todesfälle; abnehmendes Risikoverhalten, aber kein Nachweis tieferer Serokonversionsraten bei den Benutzern;

- Wright und Tompkins 2004 [41]: Auswertung 15-jähriger Erfahrung, bei jährlich ca. 1 Million Injektionen in Injektionsräumen praktisch keine Todesfälle oder schwerwiegenden Komplikationen, für viele Benutzer wird zum ersten Mal ein Kontakt mit Beratung und Hilfsangeboten hergestellt.

Strategie der Mortalitäts- und Morbiditätsprophylaxe bei Freizeitdrogenkonsum

\section{Zielsetzungen}

Folgende Ziele sollen verfolgt werden:

- Risiken beim Konsum von unbekannten Drogen und beim Mehrfachkonsum von Suchtmitteln sollen vermindert werden durch Beratung und Pillentests vor Ort;

- akute Todesfälle durch Ecstasy sollen verhindert werden durch ein geeignetes Angebot an alkoholfreien Getränken und Ruheräumen (chill-out room);

- Verkehrsunfällen auf dem Nachhauseweg soll durch Beratung vorgebeugt werden. 
Erfahrungen betreffen die folgenden Aspekte (nach EMCDDA 2001 [42]):

- das Drogentesting erleichtert eine Beratung von sonst nicht erreichbaren Konsumenten und

- Konsumtrends können rascher erkannt werden.

Diese Erfahrungen sind bislang nicht ausreichend wissenschaftlich belegt. Allerdings gibt es auch keine Belege für nachteilige Auswirkungen, wie etwa Zunahme des Konsums aufgrund des Drogentest-Angebots. Bedenken bestehen wegen einer Scheinsicherheit beim Drogentesting wegen unzureichender Untersuchungstechnik [43].

\section{Strategien risikoarmen Sexualkontakts}

\section{Zielsetzung}

Die Übertragung von Infektionskrankheiten durch penetrierenden Geschlechtsverkehr soll durch Hebung des Risikobewusstseins, durch einfachen und nicht diskriminierenden Zugang zu Kondomen und deren zweckmäßigen Gebrauch verhindert werden. Dies soll auch in Milieus erreicht werden, wo kein Geschlechtsverkehr erlaubt oder erwünscht ist (Gefängnisse, Anstalten, Heime).

Ausgewertete Erfahrungen betreffen die folgenden Aspekte:

- Safer Sex-Strategien sind generell weniger erfolgreich als die Safer Use-Strategien bei Drogenkonsumenten [44];

- Risikobewusstsein und die Erhältlichkeit von Kondomen reichen nicht aus, um sich und andere konsequent zu schützen [45];

- kulturspezifische Abneigung gegen Kondomgebrauch ist oft stärker als das Risikobewusstsein [46, 47].

Aus der prospektiven Kohortenstudie DATOS wird berichtet, dass Drogenabhängige in ambulanten Therapieeinrichtungen nicht nur ihr Risikoverhalten bezüglich Injektionen, sondern auch in Bezug auf Sexualkontakte deutlich reduzierten [48]. Dasselbe wird aus stationären ausstiegsorientierten Behandlungen berichtet [49], und ähnliches wurde in einer prospektiven Studie an homosexuellen Drogenabhängigen beobachtet [50]. Ein besonders hohes Infektionsrisiko besteht bei wechselnder Sexualpartnerschaft. Eine Studie an i. v. Drogenkonsumenten in San Francisco fand, dass Männer mit homosexuellen Kontakten hochsignifikant häufiger eine Serokonversion für HIV-1 aufwiesen als Heterosexuelle und dass Frauen mit wechselnden Sexpartnern signifikant häufiger seropositiv wurden als Frauen mit festen Partnern [51]. Eine Studie an nicht in Behandlung stehenden holländischen Drogenabhängigen wies nach, dass die Bereitschaft, Kondome zu benutzen, generell selten ist, insbesondere im Verkehr mit festen Partnern. Als Prädiktor für Kondomgebrauch, auch im Verkehr mit wechselnden Partnern, wurde als einziger Faktor „Selbstwirksamkeit“ identifiziert [52].

Daraus ergeben sich Hinweise auf spezielle Beratungsansätze. Eine Evaluation des „NIDA standard HIV testing and counselling protocol“, das bei nichtbehandelten Kokainkonsumenten zur Anwendung kam, ergab eine Reduktion des Crackkonsums, der Injektionen, der Sexpartner, aber keine Zunahme des Kondomgebrauchs [53]. Alkoholprobleme andererseits sind bei Dro- genabhängigen signifikant häufiger mit ungeschütztem Geschlechtsverkehr assoziiert. [54].

\section{Suchtmittelersatz als Risikominderungsstrategie}

Alle Therapieansätze bei i.v. Drogenkonsumenten vermögen durch eine Verminderung der Injektionsanzahl und des Risikoverhaltens beim Injizieren sowie durch eine bessere Compliance mit der Behandlung von bestehenden Infektionskrankheiten risikomindernd zu wirken. Von besonderem Interesse sind hier die Suchtersatztherapien für Heroinabhängige, da sie mittlerweile weltweit den relativ größten Anteil Abhängiger erreichen. Im Vordergrund stehen die vielfach wissenschaftlich untersuchten methadongestützten Behandlungen; an weiteren Substitutionsmitteln sind Buprenorphin und in einigen Ländern auch Diacetylmorphin (pharmazeutisches Heroin) im Gebrauch und evaluiert.

Der wichtigste schadensmindernde Aspekt ist die Reduktion des Mortalitätsrisikos. Mortalitätsraten bei Methadonsubstituierten im Vergleich zu Unbehandelten liegen bei 1:4 bis 1:3 [55-58]. Ein weiterer Hinweis ist eine signifikant erhöhte Mortalität durch Überdosis nach Abschluss der Therapie [59], die nach Wiederaufnahme einer Behandlung erneut abnimmt [60]. Allerdings besteht ein erhöhtes Mortalitätsrisiko zu Beginn einer Substitution $[61,62]$.

Eine Übersichtsarbeit auf der Basis des internationalen Forschungsstands ergab für Substitutionstherapien allgemein [63]: - signifikante Reduktion des illegalen und nicht verschriebenen Substanzkonsums mit dessen Risiken,

- verbesserter Gesundheitszustand,

- Schutz vor HIV-Infektion,

- Chancen für soziale Integration und signifikante Reduktion der Delinquenz sowie

- weniger „public nuisance“.

Insbesondere wird im Rahmen dieser Ergebnisse eine Stabilisierung des Risikoverhaltens geltend gemacht:

- weniger Verwendung gebrauchten Spritzenmaterials [64],

- weniger Injektionen [65, 66],

- signifikant weniger Serokonversionen im Vergleich zu Unbehandelten $[67,68]$ und

- nachhaltige Effekte auch nach Abschluss der Behandlung [69].

Voraussetzungen für ein gutes Ergebnis sind eine ausreichende Dosierung des Substitutionsmittels $[63,70,71]$ sowie eine ausreichende Therapiedauer $[64,68,72,73]$.

Eine weitere schadensmindernde Wirkung besteht in der besseren Compliance mit einer antiretroviralen Medikation und damit einer besseren Therapieprognose und Lebensverlängerung [74] sowie in einer verlangsamten Krankheitsprogression der HIV-Infektionen [75]. Aufgrund einer Übersicht über 33 Studien, publiziert zwischen 1988 und 1998, die sich vor allem auf Methadonbehandlungen beziehen, gilt diese Behandlung als eindeutige schadensmindernde Maßnahme im Sinne einer Reduktion von „needle sharing“ und HIV-Risiko [76]. 
Anhand einer Studie zur Reduktion von HIV-Infektionen im Zusammenhang mit einer Vermehrung von Methadonprogrammen wurde außerdem ein positiver Kosten-Nutzen-Effekt errechnet [77]. Die positiven Ergebnisse werden nicht als ein Resultat der Substitutionsmedikation allein verstanden. Vielmehr ist die Qualität der Begleitbehandlung und -betreuung ein ebenso wichtiger Faktor für den Verbleib in der Therapie und für deren Erfolg [78, 79]. Bei so genannter niederschwelliger Methadonsubstitution (ohne verbindliche Begleitbehandlung, ohne Urinkontrollen) gelten die erwähnten Resultate nur bedingt. Nach niederländischer Erfahrung bildet sie bei niedriger Dosierung keinen Schutz vor Erwerb oder Weitergabe einer HIV- oder Hepatitisinfektion $[45,80]$. Eine Überleitung zu einem strukturierten Programm gelingt eher selten. Andererseits kann bei sozial gut stabilisierten Patienten sekundär ein niederschwelliges Regime mit Verzicht auf die sonst obligate Begleitbetreuung einen Therapieabbruch verhindern [81].

Eine schadensmindernde Substitutionstherapie gibt es außer bei Opiatabhängigkeit auch bei Nikotinabhängigkeit. Mit Nikotinersatzpräparaten (Kaugummi, Patch), bei denen die Schadstoffe des Tabakrauchs wegfallen, lässt sich Folgendes erreichen:

- eine Reduktion der Menge gerauchter Zigaretten [82];

- Unterstützung beim Aufhören mit Rauchen (Shiffman et al. 2002 [83]: 23\% Abstinenz nach zehn Wochen, Glover et al. [85]: 18\% Abstinenz nach 12 Monaten);

- die besten Resultate werden in Kombination mit psychosozialer Beratung erzielt [86], positive Resultate bei Selbstbehandlung ohne Begleitung gelten als zu wenig gesichert [87];

- bessere Abstinenzergebnisse bei längerfristiger Nikotinersatztherapie [88];

- das Risiko eines unerwünschten chronischen Gebrauchs der Ersatzmittel wird mit $0,4 \%$ nach 24 Monaten als gering erachtet [89].

\section{Unterstützende Maßnahmen einer Risikominderungspolitik}

Eine Reihe weiterer Maßnahmen soll die Ziele einer Schadensminderung unterstützen. Dazu gehören niederschwellige Kontakt- und Anlaufstellen sowie medizinische Hilfseinrichtungen für Suchtmittelkonsumenten, Hilfsprogramme für sich prostituierende Personen (Sexworkers sowie Gelegenheitsprostituierte), Notschlafstellen, betreute Wohnmöglichkeiten mit Betreuungsangebot, Tagesprogramme und Arbeitseinsätze mit Beratungsangebot sowie Straßenarbeit. Derartige Einrichtungen vermindern sowohl Verelendungszustände bei Betroffenen als auch Belastungen der Bevölkerung. Ein gemeinsames Merkmal bildet der Verzicht auf eine Diskriminierung des fortgesetzten Suchtmittelkonsums, aber keine Toleranz für Gewalt und Drogenhandel in den Einrichtungen. Erreichung der Zielgruppe und zweckmäßiges Arbeiten setzen klare Absprachen mit den lokalen Behörden und insbesondere mit Polizeidienststellen voraus.

Erfahrungen mit derartigen Einrichtungen sind ebenfalls dokumentiert. In Stichworten: weniger Belästigung der Bevölkerung, bessere Akzeptanz für Hilfsangebote und Therapien seitens der Betroffenen, weniger Abhängige ohne jeden Kontakt mit der Helferszene sowie rasche Erkennung neuer Trends beim Drogenkonsum und seinen Auswirkungen [84].
Widerstände und Befürrchtungen

Trotz positiver Erfahrungen gibt es nach wie vor eine weit verbreitete Ablehnung der geschilderten schadensmindernden Maßnahmen. Die Befürchtungen sind vielfältiger Natur:

- Nachteile für die Prävention durch „Verharmlosung“ des Suchtmittelkonsums;

- Erhöhung von Konsumbereitschaft und Konsum;

- Verminderung des Risikobewusstseins und Einladung zu mehr Risikoverhalten;

- Unvereinbarkeit mit den UNO-Konventionen;

- Schritt in Richtung auf das (versteckte) Ziel einer Drogenlegalisierung.

Allerdings konnte für keine dieser Befürchtungen ein wissenschaftlicher Nachweis erbracht werden. Am Beispiel Heroinkonsum ist ersichtlich, dass in Ländern mit ausgebauten schadensmindernden Maßnahmen und Substitutionstherapien einschließlich heroingestützter Behandlung (Niederlande, Schweiz) dieser Konsum sich ebenso stabilisierte oder sogar reduzierte wie in Ländern ohne einen solchen Ausbau. Dass Schadensminderung mit den UNO-Konventionen vereinbar ist, bestätigte die zuständige Aufsichtsbehörde [90]. Eine grundsätzliche Opposition stellt der Schadensminderung das Ziel der Konsumminderung entgegen. Eine Analyse dieser unterschiedlichen Zielsetzungen kommt zum Schluss, dass die Vermeidung nachteiliger Folgen von Konsum, Produktion und Kontrolle von Suchtmitteln Vorrang haben muss und dass Reduktion des Konsums eine Strategie unter anderen darstellt, dieses Ziel zu erreichen [91].

\section{Wege zur Optimierung}

Eine Wirksamkeit der Maßnahmen in signifikantem Ausmaß ist in vielen Fällen durch randomisierte Studien abgesichert. Trotzdem gibt es Grenzen der Wirksamkeit, infolge einer begrenzten Erreichung der Zielgruppen, unzulänglicher Ausstattung und Kompetenz der Einrichtungen, mangelhafter Konzertierung mit therapeutischen und polizeilichen Maßnahmen, gesetzlicher Schranken und fehlender politischer Unterstützung.

Angesichts solcher Grenzen sind Anstrengungen in den folgenden Bereichen erforderlich: Training und Supervision der Mitarbeiterteams, benutzerfreundliche Atmosphäre der Einrichtung, klare und transparente Regeln für Benutzer, Schutz der Nachbarschaft vor Immissionen, klare Vereinbarungen mit der Polizei, Zusammenarbeit mit medizinischen und sozialen Diensten, Informationsarbeit für eine weitere Öffentlichkeit und politische Unterstützung auf allen Ebenen. Dazu gehören im Weiteren - im Interesse einer Optimierung und Anpassung an veränderte Verhältnisse - eine Dokumentierung des Geleisteten sowie die Registrierung erwünschter und unerwünschter Auswirkungen. Und schließlich soll Schadensminderung ergänzt werden durch primär- und sekundärpräventive Ansätze und durch ausreichende und gut zugängliche Behandlungsangebote, um eine optimale Wirkung zu haben [92]. 
Integration in eine umfassende Suchtmittelpolitik

Ein frühes Beispiel für eine solche Integration ist die Nationale Drogenpolitik der Schweiz von 1991. Im Rahmen dieser so genannten Vier-Säulen-Politik stehen Maßnahmen der Schadensminderung gleichwertig neben präventiven, therapeutischen und repressiven Maßnahmen. Gleichzeitig wurde der Grundsatz festgehalten, diese Maßnahmen auf ihre Tragfähigkeit und Wirkungen hin zu evaluieren und weiter zu entwickeln, im Sinne einer evidenzgeleiteten Politik. In nationalen Volksabstimmungen wurde diese Politik wiederholt mit Mehrheiten bestätigt, mit klarer Ablehnung zweier Initiativen einer einseitig repressiven Politik als auch einer Legalisierung der verbotenen Substanzen.

Anhand einer Reihe von Indikatoren ist ablesbar, dass sich seither die Drogensituation in der Schweiz in folgender Hinsicht verbessert hat [93]:

- seit 1992 sinkende Anzahl von Drogentodesfällen (1992: 419; 2001: 197);

- sinkende Anzahl der HIV-Infektionen bei Drogenabhängigen (1989: 833; 2001: 97);

- steigendes Durchschnittsalter Heroinabhängiger in Polizeiund Therapiestatistiken; - schlechtes Image des Heroins und geringe Neigung zu Experimentierkonsum mit Heroin bei Jugendlichen.

Außerdem war ein signifikanter Rückgang der typischen drogenbedingten Delinquenz festzustellen $[94,95]$. Neue Herausforderungen im Bereich von Schadensminderung und Therapie sind Mehrfachabhängigkeiten, Dualdiagnosen sowie eine neue Lust an Risikoverhalten und Rauschtrinken bei Minderjährigen.

\section{Fazit}

Aufgrund dieser Übersicht darf festgehalten werden:

- Maßnahmen einer Risikominderung und ihre Vereinbarkeit im Rahmen einer umfassenden Drogenpolitik sind machbar und akzeptierbar;

- Risikominderung bedeutet Schadensminderung; ihre Ziele sind erreichbar;

- Schadensminderung steht nicht im Gegensatz zu Prävention und Therapie; die Ansätze lassen sich miteinander verbinden;

- negative Auswirkungen sind kaum belegt, aber Verbesserungen und Anpassungen an neue Herausforderungen sind nötig.

\section{Literatur}

${ }^{1}$ Miller M, Eskild A, Mella I et al. Gender differences in stringe exchange programs use in Oslo, Norway. Addiction 2001; 96: 1639-1651

2 Longshore D, Bluthenthal RN, Stein MD. Needle exchange programme: attendance and injection risk in Providence. AIDS Education Prevention 2001; 13: $78-90$

${ }^{3}$ des Jarlais DC, Friedman P, Grund JP et al. HIV risk behaviour among participants of syringe exchange programmes in Central/Eastern Europe and Russia. Internat J Drug Policy 2002; 13: 165 - 170

${ }^{4}$ Rutter S, Dolan K, Wodak A et al. Prison syringe exchange: a review of international research and program development. Sydney: National Drug and Alcohol Research Centre, 2001
${ }^{5}$ Stöver H, Nelles J. Ten years of experience with needle and syringe exchange programmes in European prisons. International J Drug Policy 2003; 14: 437-444

${ }^{6}$ Dolan K, Rutter S, Wodak A. Prison-based syringe exchange programmes: a review of international research and development.Addiction 2003; 98: 153-158

${ }^{7}$ Heinemann A, Gross U. Prevention of bloodborne virus infections among drug users in an open prison by syringe vending machines. Sucht 2001; 47: 57-65

8 des Jarlais DC, Friedman SR, Ward TP. Harm reduction: a public health response to the Aids epidemic among injecting drug users. Annual Review Public Health 1993; 14: $413-450$

${ }^{9}$ Lurie P, Reingold AL. The public health impact of needle exchange programs in the United States and abroad. San Francisco: Institute for Health Policy Studies, 1993

${ }^{10}$ Gibson DR, Flynn NM, Perales D. Effectiveness of syringe exchange programs in reducing HIV risk behavior and HIV seroconversion among injecting drug users. AIDS 2001; 15: 1329-1341

${ }^{11}$ Doherty MC, Junge B, Rathouz B et al. The effect of a needle exchange program on numbers of discarded needles: a 2-year follow-up. Amer J Public Health 2000; 90: 936-939

12 Broadhead RS, Kerr TH, Grund JPC et al. Safer injection facilities in North America: their place in public policy and health initiatives. J Drug Issues 2002; 32: 329-356

${ }^{13}$ MacDonald M, Law M, Kaldor J et al. Effectiveness of needle and syringe programmes for preventing HIV transmission. Internat J Drug Policy 2003; $14: 353-357$

${ }^{14}$ Kuo I, Brady J, Butler C et al. Feasibility of referring drug users from a needle exchange program into an addiction treatment program: experience with a mobile treatment van and LAAM maintenance. J Substance Abuse Treatment 2003; 24: 67-74

${ }^{15}$ Bluthenthal RN, Gogineni A, Longshore D et al. Factors associated with readiness to change drug use among needle-exchange users. Drug Alcohol Dependence 2001; 62: 225-230

${ }^{16}$ Riley ED, Safaeian M, Strathdee SA et al. Drug user treatment referrals and entry among participants of a needle exchange programme. Substance Use Misuse 2002; 37: 1869-1886

17 MacMaster SA, Vail KA. Demystifying the injection drug user: willingness to participate in traditional drug treatment services among participants in a needle exchange program. J Psychoactive Drugs 2002; 34: $289-294$

${ }^{18}$ Hagan H, McGough JP, Thiede H et al. Reduced injection frequency and increased entry and retention in drug treatment associated with needle-exchange participation in Seattle drug injectors. J Substance Abuse Treatment 2000; 19: 247-252

${ }^{19}$ Stein MD, Charuvastra A, Maksad J et al. A randomized trial of a brief alcohol intervention for needle exchangers. Addiction 2002; 97: $691-700$

20 Stein MD, Charuvastra A, Anderson B et al. Alcohol and HIV risk taking among intravenous drug users. Addictive Behaviors 2002; 27: $727-736$

${ }^{21}$ Baker A, Boggs TG, Lewin TJ. Randomised controlled trial of brief cognitive-behavioural interventions among regular users of amphetamine. Addiction 2001; 96: 1279-1287

${ }^{22}$ Levounis P, Galanter M, Dermatis H et al. Correlates of HIV transmission risk factors and considerations forinterventions in homeless, chemically addicted and mentally ill patients. J Addictive Diseases 2002; 21: $61-72$

${ }^{23}$ Aitken C, Moore D, Higgs P et al. The impact of a police crackdown on a street drug scene: evidence from the street. Intern J Drug Policy 2002; 13: $189-198$

${ }^{24}$ Bluthenthal RN, Malik MR, Grau LE et al. Sterile syringe access conditions and variations in HIV risk among drug injectors in three cities. Addiction 2004; 99: 1136-1146

${ }^{25}$ McVeigh J, Beynon C, Bellis MA. New challenges for agency based syringe exchange schemes: analysis of 11 years of data (1991-2001) in Merseyside and Cheshire, United Kingdom. Internat J Drug Policy 2003; 14: 399-405

${ }^{26}$ Moatti JP, Vlahov D, Feroni I et al. Multiple access to sterile syringes for injection drug users: vendine machines, needle exchange programs and legal pharmacy sales in Marseille, France. Europ Addiction Research 2001; 7: 40-45

${ }^{27}$ Wood E, Tyndall MW, Spittal PM et al. Needle exchange and difficulty with needle access during an ongoing HIV epidemic. Internat J Drug Policy 2002; 13: 95 - 102 
${ }^{28}$ Avants SK, Warburton LA, Hawkins KA et al. Continuation of high-risk behavior by HIV-positive drug users' treatment implications. J Substance Abuse Treatment 2002; 19: 15-22

${ }^{29}$ Caflisch C, Wang J, Zbinden R. The role of syringe filters in harm reduction among injecting drug users. Amer J Public Health 1999; 89: $1252-1254$

${ }^{30}$ Grund JPC, Kaplan CD, Adriaans NFP et al. Drug sharing and HIV transmission risks: the practice of "frontloading“ in the Dutch injecting drug user population. J Psychoactive Drugs 1999; 23: 1-10

${ }^{31}$ WHO. Effectiveness of sterile needle and syringe programming in reducing HIV/AIDS among injecting drug users. Geneva: World Health Organisation, 2004

32 DHHS. U.S. Department of Health and Human Services. Bulletin, April 1993

${ }^{33}$ Hughes RA. Drug injectors and the cleaning of needles and syringes. Europ Addiction Research 2000; 6: 20-30

${ }^{34}$ Hedrich D. European report on drug consumption rooms. Lisbon: European Monitoring Centre for Drugs and Drug Addiction EMCDDA, 2004

${ }^{35}$ Kerr T, Wood E, Palepu A et al. Responding to an explosive HIV epidemic driven by frequent cocaine injection: is there a role for safe injecting facilities? J Drug Issues 2003; 33: 579-608

36 Ronco C, Spuhler G, Coda P et al. Evaluation der Gassenzimmer I, II und III in Basel. Sozial Präventivmedizin 1996; 41 (Suppl 1): 58 - 68

${ }^{37} \mathrm{Kemmesis} U$. The open drug scene and the safe injection room offers in Frankfurt am Main: final report. Münster: Indro, 1999

${ }^{38}$ Dolan K, Kimber J, Fry C et al. Drug consumption facilities in Europe and the establishment of supervised injecting centres in Australia. Drug Alcohol Review 2000; 19: 337-346

${ }^{39}$ Happel V. Konsumräume. Eine effektive Maßnahme zur Schadensminderung bei DrogengebraucherInnen und BürgerInnen. Akzeptanz 2000; 8: 30-36

${ }^{40}$ Fischer B, Rehm J, Kim G et al. Safer injecting facilities (SIFs) for injection drug users in Canada: a review and call for an evidencefocused pilot trial. Canadian J Public Health 2002; 93: 336- 338

${ }^{41}$ Wright NMJ, Tompkins CNE. Supervised injecting centres. Brit Med J 2004; 328: $100-102$

42 EMCDDA . On-site pill-testing interventions in the European Union. Lisbon: European Monitoring Centre for Drugs and Drug Addiction, 2001

${ }^{43}$ Winstock AR, Wolff K, Ramsey J. Ecstasy pill testing: harm minimization gone too far? Addiction 2001; 96: 1139-1148

44 des Jarlais DC, Friedman SR. HIV and intravenous drug use. AIDS 1988; 2 (Suppl 1): S65-S69

${ }^{45}$ Hartgers C, van den Hoeck A, Krijnen P et al. HIV prevalence and risk behavior among injecting drug users who participate in „lowthreshold" methadone programs in Amsterdam. Amer J Public Health 1992; 82: 547-551

${ }^{46}$ Amadora-Nolasco F, Alburo RE, Aguilar EJT et al. Knowledge and perception of risk for HIV and condor use among male injecting drug users in Cebu city, Phillipines. Drug Alcohol Review 1992; 21: $137-143$

47 Johnson RA, Gerstein GR, Pack A et al. HIV risk behaviors in AfricanAmerican drug injectors networks: implications of injection-partner mixing and partnership characteristics. Addiction 2002; 97: $1011-1024$

${ }^{48}$ Broome KM, Joe GW, Simpson DD. HIV risk reduction in outpatient drug abuse treatment: individual and geographic differences. AIDS Education Prevention 1999; 11: 293-306

${ }^{49}$ Jainchill N, Yagelka J, Hawke J et al. Adolescent admissions to residential drug treatment: HIV risk behaviours pre- and posttreatment Psychology Addictive Behaviors 1999; 13: 163 - 173

50 Stall RD, Paul JP, Barrett DC et al. An outcome evaluation to measures changes in sexual risk-taking among gay men undergoing substance use disorder treatment. J Studies Alcohol 1999; 60: 837-845

${ }^{51} \mathrm{Kral} \mathrm{AH}$, Bluthenthal RN, Lorwick J et al. Sexual transmission of HIV-1 among injection drug users in San Francisco, USA: risk-factor analysis. Lancet 2001; 357: 1397-1401

52 van Empelen P, Schaalma HP, Kok G et al. Predicting condom use with casual and steady sex partners among drug users. Health Education Research 2001; 16: 293-305

53 Compton WM, Cottler LB, Ben-Abdallah A et al. The effects of psychiatric comorbidity on response to a HIV prevention intervention. Drug Alcohol Dependence 2000; 58: 247-257
${ }^{54}$ Ehrenstein V, Horton NJ, Samet JH. Inconsistent condom use among HIV-infected patients with alcohol problems. Drug Alcohol Dependence 2004; 73: 159-166

55 Caplehorn JRM, Dalton MSY, Cluff MC et al. Retention in methadone maintenance and heroin addict's risk of death. Addiction 1994; 89: $203-207$

${ }^{56}$ Raschke P, Püschel K, Heinemann A. Rauschgiftmortalität und Substitutionstherapie in Hamburg. Suchttherapie 2000; 1: 43-48

57 Mattick R, Degenhardt L. Methadone-related and heroin-related deaths among opiate users: methadone helps save lives. Addiction 2003; 98 (4): 419-425

${ }^{58}$ Stenbacka M, Leifman A, Romelsjo A. The impact of methadone on consumption of inpatient care and mortality, with special reference to HIV status. Subst Use Misuse 1998; 33 (14): 2819-2834

${ }^{59}$ Langendam MW, van Brussel GH, Coutinho RA et al. The impact of harm-reduction-based methadone treatment on mortality among heroin users. Am J Public Health 2001; 91 (5): 774 - 780

${ }^{60}$ Grönbladh L, Ohland LS, Gunne LM. Mortality in heroin addiction: impact of methadone treatment. Acta Psychiat Scand 1990; 82: 223 - 227

61 Zador DA, Sunjic SD. Methadone-related deaths and mortality rate during induction into methadone maintenance, New South Wales, 1996. Drug and Alcohol Review 2002; 21: 131-136

62 Wagner-Servais D, Erkens M. Methadone-related deaths associated with faulty induction procedures. Journal of Maintenance in the Addictions 2003; 2: $57-67$

${ }^{63}$ Ward J, Mattick RP, Hall W. The effectiveness of methadone maintenance treatment: HIV and infectious hepatitis. In: Ward J, Mattick RP, Hall W (Hrsg). Methadone maintenance: treatment and other opioid replacement therapies. Amsterdam: Harwood, 1995: 59-73

${ }^{64}$ Marsch LA. The efficacy of methadone maintenance interventions in reducing illicit opiate use, HIV risk behavior and criminality: a metaanalysis. Addiction 1998; 93 (4): 515 - 532

${ }^{65}$ Caplehorn JRM, Ross M. Methadone maintenance and the likelihood of risky needle sharing. Internat J Addictions 1995; 30: 685-698

${ }^{66}$ Gowing L, Farrell M, Bornemann R et al. Substitution treatment of injecting opioid users for prevention of HIV infection. Oxford: The Cochrane Library, 2004

${ }^{67}$ Metzger DS, Woody GE, McLellan AT. Human Immunodeficiency Virus (HIV) seroconversion among intravenous drug users in- and out-oftreatment: an 18-month prospective follow-up. Journal of Acquired Immune Deficiency Syndromes 1993; 6: 1049-1056

${ }^{68}$ Moss AR, Vranizan K, Gorter R et al. HIV seroconversion in intravenous drug users in San Francisco 1985 - 1990. AIDS 1994; 8: 223 - 231

${ }^{69}$ Gossop M, Marsden J, Steward D et al. The National Treatment Outcome Research Study (NTORS): 4-5 year follow-up results. Addiction 2003; 98: 291 - 303. Gossop M, Marsden J, Stewart D et al. Reduction or cessation of injecting risk behaviours? Treatment outcomes at 1-year follow-up. Addictive Behaviors 2003; 28: 785 - 793

70 Ball JC, Ross A. The effectiveness of methadone maintenance treatment. New York: Springer, 1991

71 Serpelloni G, Carrieri MP, Rezza G et al. Methadone treatment as a determinant of HIV risk reduction among injecting drug users: a nested case control study. AIDS Care 1994; 16: 215-220

${ }^{72}$ Chaisson RE, Bacchetti P, Osmond D et al. Cocaine use and HIV infection in intravenous drug users in San Francisco. Journal of the American Medical Association 1989; 261: 561-565

${ }^{73}$ Schoenbaum EE, Hartel D, Selwyn PA et al. Risk factors for human immunodeficiency virus infection in intravenous drug users. New England Journal of Medicine 1989; 321: 874-879

${ }^{74}$ Avants SK, Margolin A, Warburton LA et al. Predictors of nonadherence to HIV-related medication regimens during methadone stabilisation. American Journal on Addictions 2001; 10: 69-78

${ }^{75}$ Weber R, Ledergerber B, Opravil M et al. Progression of HIV infection in misusers of injected drugs who stop injecting or follow a programme of maintenance treatment with Methadone. British Medical Journal 1990; 301: $1362-1365$

${ }^{76}$ Sorenson L, Copeland AM. Drug abuse treatment as an HIV prevention strategy: a review. Drug Alcohol Dependence 2000; 59: 17-31

77 Zaric GS, Barnett PG, Brandeau ML. HIV transmission and the cost-effectiveness of methadone maintenance. Amer J Public Health 2000; 90: $1100-1111$

${ }^{78}$ McLellan AT, Arndt IO, Woody GE. Psychosocial services in substance abuse treatment? A dose-ranging study of psychosocial services. J Am Med Assn 1993; 269 (15): 1953-1959 
${ }^{79}$ McLellan AT. The role of psychosocial services in drug abuse treatment. In: Waal H, Haga E (Hrsg). Maintenance treatment in heroin addiction. Oslo: Cappelen, 2003: 267-295

${ }^{80}$ van Ameijden EJC, van den Hoeck AAR, van Haastrecht HJA et al. The harm reduction approach and risk factors for Human Immunodeficiency Virus (HIV) seroconversion among injecting drug users in Amsterdam. Amer J Epidemiology 1992; 136: 236 - 243

${ }^{81}$ Raschke P. Substitutionstherapie. Ergebnisse langfristiger Behandlung von Opiatabhängigen. Freiburg im Breisgau: Lambertus, 1994

${ }^{82}$ Fagerström KO, Hughes JR. Nicotine concentrations with concurrent use of cigarettes and nicotine replacement: a review. Nicotine Tobacco Research 2002; 4 (Suppl 2): $73-79$

83 Shiffman S, Gorsline J, Gorodetzky CW. Efficacy of over the counter nicotine patch. Nicotine Tobacco Research 2002; 4: 477-483

${ }^{84}$ BAG (Hrsg). Evaluationen des BAG im Suchtbereich 1990-2000. Bern: Bundesamt für Gesundheit, 2002

85 Glover ED, Glover PN, Franzon M et al. A comparison of a nicotine sublingual tablet and placebo for smoking cessation. Nicotine Tobacco Research 2002; 4: $441-450$

${ }^{86}$ Hughes JR, Goldstein MG, Hurt RD. Recent advances on the pharmacotherapy of smoking. JAMA 1999; 281: $72-81$
${ }^{87}$ Walsh RA, Penman AG. The effectiveness of nicotine replacement therapy over-the-counter. Editorial Drug Alcohol Review 2000; 19: $243-248$

${ }^{88}$ Batra A, Buchkremer G. Die Dauer der Nikotinersatztherapie. Ein Prädiktor für die langfristige Abstinenz? Sucht 2000; 46: 414-423

${ }^{89}$ Shiffman S, Hughes JR, Pillitteri JL et al. Persistent use of nicotine replacement therapy: an analysis of actual purchase patterns in a population based sample. Tobacco Control 2003; 12: 310-316

${ }^{90}$ International Narcotics Control Board (INCB). Annual Report 2004

${ }^{91}$ Caulkins JP, Reuter P. Setting goals for drug policy: harm reduction or use reduction? Addiction 1997; 92: $1143-1150$

92 MacCoun RJ. The psychology of harm reduction: comparing alternative strategies for modifying high-risk behaviors. Wellness Lectures 1996; 6: 5-27

${ }^{93}$ Vogt R. Aktuelle Situation, Entwicklungen und Herausforderungen der Schadensverminderung. Abhängigkeiten 2002; 8: 5-17

${ }^{94}$ Killias M, Lamon P, Clerici C et al. Tendances de la criminalité en Suisse de 1984 à 2000. Risques objectifs et perceptions subjectives. Lausanne: Institut de Police Scientifique et de Criminologie, 2000

${ }^{95}$ Killias M. Heroinverschreibung. Ein erfolgreiches Programm zur Kriminalitätsprävention. Abhängigkeiten 2004; 10: 16 -18 